OPEN ACCESS

Edited by:

Mattias Collin

Lund University, Sweden

Reviewed by:

Timothy J. Foster,

Trinity College Dublin, Ireland Julius Nyalwidhe,

Eastern Virginia Medical School,

United States

*Correspondence:

Marc Burian

mburian@ukaachen.de

tThese authors have contributed equally to this work and share senior authorship

Specialty section:

This article was submitted to Infectious Agents and Disease,

a section of the journal

Frontiers in Microbiology

Received: 22 June 2021 Accepted: 01 September 2021 Published: 21 September 2021

Citation:

Burian M, Plange J, Schmitt L, Kaschke A, Marquardt $Y$, Huth L, Baron JM, Hornef MW, Wolz C and

Yazdi AS (2021) Adaptation of Staphylococcus aureus to the Human Skin Environment Identified

Using an ex vivo Tissue Model.

Front. Microbiol. 12:728989. doi: 10.3389/fmicb.2021.728989

\section{Adaptation of Staphylococcus aureus to the Human Skin Environment Identified Using an ex vivo Tissue Model}

\author{
Marc Burian ${ }^{1 *}$, Johanna Plange ${ }^{1}$, Laurenz Schmitt ${ }^{1}$, Anke Kaschke ${ }^{1}$, Yvonne Marquardt ${ }^{1}$, \\ Laura Huth $^{1}$, Jens M. Baron ${ }^{1}$, Mathias W. Hornef ${ }^{2}$, Christiane Wolz ${ }^{3 \dagger}$ and Amir S. Yazdi' ${ }^{1+}$ \\ ${ }^{1}$ Department of Dermatology and Allergology, RWTH University Hospital Aachen, Aachen, Germany, ${ }^{2}$ Institute of Medical \\ Microbiology, RWTH University Hospital Aachen, Aachen, Germany, ${ }^{3}$ Interfaculty Institute of Microbiology and Infection \\ Medicine, University of Tuebingen, Tuebingen, Germany
}

The healthy human epidermis provides physical protection and is impenetrable for pathogenic microbes. Nevertheless, commensal and pathogen bacteria such as Staphylococcus aureus are able to colonize the skin surface, which may subsequently lead to infection. To identify and characterize regulatory elements facilitating adaptation of $S$. aureus to the human skin environment we used ex vivo tissue explants and quantified $S$. aureus gene transcription during co-culture. This analysis provided evidence for a significant downregulation of the global virulence regulator agr upon initial contact with skin, regardless of the growth phase of $S$. aureus prior to co-culture. In contrast, the alternative sigma factor $\mathrm{B}(\mathrm{sig} \mathrm{B})$ and the antimicrobial peptide-sensing system (graRS) were expressed during early colonization. Consistently, sigB target genes such as the clumping factor $\mathrm{A}(\mathrm{clf} A)$ and fibrinogen and fibronectin binding protein A (fnbA) were strongly upregulated upon skin contact. At later timepoints of the adhesion process, wall teichoic acid (WTA) synthesis was induced. Besides the expression of adhesive molecules, transcription of molecules involved in immune evasion were increased during late colonization (staphylococcal complement inhibitor and staphylokinase). Similar to nasal colonization, enzymes involved in cell wall metabolism (sceD and at/A) were highly transcribed. Finally, we detected a strong expression of proteases from all three catalytic classes during the entire colonization process. Taken together, we here present an ex vivo skin colonization model that allows the detailed characterization of the bacterial adaptation to the skin environment.

Keywords: ex vivo skin explant, proteases, global regulators, accessory gene regulator (agr), bacterial adhesion, immune evasion, colonization, human skin (in vivo)

\section{INTRODUCTION}

Healthy skin protects from microbial colonization and infection through a variety of mechanisms such as a competitive microbiota and a large number of antimicrobial and immunological effector molecules including lipids, salts, enzymes, and an acidic surface pH (Chen et al., 2018). Under diseased conditions, promoted by changes in the microbiota composition, deficiency of skin barrier 
components and loss of skin barrier integrity, pathogens such as Staphylococcus aureus can cause severe skin and soft tissue infections (Olaniyi et al., 2017). Besides these infections, $S$. aureus colonizes lesional skin of atopic dermatitis (AD) patients especially during an eczema flare. Here the diversity of the normal microflora is drastically reduced and the increased abundance of $S$. aureus is linked to the severity and worsening of this chronic inflammatory skin disease (Totte et al., 2016). Beside its primary ecological niche-the human nose-the skin represents a second important reservoir of S. aureus in AD patients (Alsterholm et al., 2017). In contrast to atopic skin, only $\sim 10-20 \%$ of healthy subjects are colonized with $S$. aureus on the skin (Matsui et al., 2000; Shi et al., 2016). Only limited information is available on $S$. aureus factors required to colonize the healthy skin and the concomitant expression of putative virulence factors. It is likely that some of these factors are induced upon contact of $S$. aureus with the skin surface or by specific skin micronutrients or environmental factors. $S$. aureus is equipped with several interactive regulatory systems, which orchestrate appropriate gene expression during colonization and infection (Jenul and Horswill, 2019). The information on direct ex vivo or in vivo bacterial gene expression is so far limited. We recently characterized $S$. aureus gene expression during persistent nose colonization of healthy volunteers (Burian et al., 2010a,b, 2012) and during the initial phase of nasal colonization in cotton rats (Burian et al., 2010a). From these analyses it became evident that the global virulence regulators (agr, sae, sigB, graRS) were not activated during colonization, whereas expression of the essential two-component regulatory system walKR was increased compared to in vitro grown bacteria. Furthermore, nasal $S$. aureus colonization was characterized by the expression of genes mediating adhesion ( clf $\mathrm{B}, f n b \mathrm{~A}$, isd $\mathrm{A}, \operatorname{tag} \mathrm{O})$, cell surface dynamics/remodeling (sce $\mathrm{D}$, atl $\mathrm{A}, \operatorname{oat} \mathrm{A})$, the expression of immune evasion genes ( $a k, c h p)$ and the lack of toxin transcription ( $h l a$ psm). We hypothesize that gene expression might differ significantly once $S$. aureus leaves its habitat in the nose and adapts to the skin environment and that such regulatory switches contribute to successful colonization. On the long-term, a better understanding of the specific gene expression pattern will give insight in the importance of single highly expressed factors and the importance of regulatory systems.

In the present study, we established an ex vivo skin explants model to investigate $S$. aureus adaption to human skin. The model is similar to a recent described model for the investigation of topical therapeutics (Neil et al., 2020). Our model allows continuous monitoring of gene expression changes up to 8 days after initial contact with $S$. aureus. Here, the colonization of healthy skin led to a uniform response of the pathogen independently of the human host and a similar gene expression pattern was observed when $S$. aureus from exponential or post exponential growth phase were inoculated. In this model which is largely deprived of immune cells, the effects of $S$. aureus adaptation by direct contact to the human skin can be determined, independently of infiltrating immune cells.

\section{MATERIALS AND METHODS}

\section{Bacterial Strains and Growth Conditions}

S. aureus USA300 JE2 (Fey et al., 2013) was grown overnight in CnT-Prime medium (CELLnTEC), diluted to an initial optical density value at $600 \mathrm{~nm}\left(\mathrm{OD}_{600}\right)$ of 0.05 in fresh CnT-Prime medium and grown with shaking $(180 \mathrm{rpm})$ at $37^{\circ} \mathrm{C}$ either to the exponential $\left(\mathrm{OD}_{600}=0.5\right)$ or post-exponential $\left(\mathrm{OD}_{600}=0.5+4\right.$ h) growth phase.

\section{Ethics Statement}

Ex vivo skin explants were obtained from skin tumor surgery in which pieces of healthy skin were removed for surgical reasons to compensate for the circular excisions upon surgical closure. This approach was approved by the local ethics committee of the Medical Faculty RWTH University of Aachen, Germany (EK 404/19). In accordance with the Declaration of Helsinki Principles guidelines written consent was obtained from all participants involved in the study.

\section{Human ex vivo Skin Model}

Immediately after surgery, subcutaneous adipose tissue was removed and skin explants were punched into defined pieces (4 $\mathrm{mm}$ in diameter) using surgical biopsy punches (pfm medical AG). Skin samples were incubated for $12 \mathrm{~h}$ in DMEM (Pan Biotech) supplemented with 10\% fetal calf serum (Pan Biotech) and $1 x$ antibiotic-antimycotic (Gibco $\left.{ }^{\mathrm{TM}} 15240062\right)$ to remove the remaining skin flora. The skin area was routinely disinfected prior to surgery and subsequent incubation in DMEM supplemented with $1 \mathrm{x}$ antibiotic-antimycotic $(12 \mathrm{~h})$ ensured a significant reduction of the natural skin flora. In order to enable colonization with $S$. aureus samples were washed twice with pre-warmed $37^{\circ} \mathrm{C}$ phosphate-buffered saline (PBS) [Pan Biotech] and placed into Millicell ${ }^{\circledR}$ 24-well cell culture inserts with a $0.4 \mu \mathrm{m}$ pore size membrane (Merck). Inserts were placed into 24-well plates prefilled with $300 \mu \mathrm{l}$ of CnTPrime medium and explants were cultured at the air-liquid interface at $37^{\circ} \mathrm{C}$ and $5 \% \quad \mathrm{CO}_{2}$. After 4 days, CnT-Prime medium was added to the wells, ensuring the continuous supply of nutrients to the tissue from below. Either exponential or post-exponential grown S. aureus USA300 JE2 bacteria were harvested by centrifugation at $4.000 \mathrm{rpm}$ for $10 \mathrm{~min}$ and resuspended in CnT-Prime medium. $9 \mu \mathrm{l}$ of bacterial suspension corresponding to $1 \times 10^{6}$ colony-forming units (CFUs) were applied step wise in 3-4 $\mu$ l steps to the surface of the skin explants.

As a control, we randomly applied the concentrated medium to blood agar plates to examine for the presence of $S$. aureus. No growth of $S$. aureus was detected in any of the plates (data not shown). For transcript analyses infected skin samples were incubated for $15 \mathrm{~min}, 1,2,4,6,18,28$, and $74 \mathrm{~h}$, as well as $5,6,7$, and 8 days at $37^{\circ} \mathrm{C}$ and $5 \% \mathrm{CO}_{2}$. At the defined times, skin explants were removed from the inserts, cut into small pieces and dissolved in $1 \mathrm{ml}$ TRIzol $^{\mathrm{TM}}$ LS reagent (Thermo Fisher Scientific) for RNA isolation. The bacterial inoculum that was applied to the skin's surface was also transferred into 
$1 \mathrm{ml}$ of TRIzol ${ }^{\mathrm{TM}}$ LS reagent. In addition, wells containing $300 \mu \mathrm{l}$ of CnT Prime medium only, were also inoculated with $1 \times 10^{6}$ CFUs of the inoculum suspension to analyze the in vitro growth without skin. Bacteria grown in medium only were also transferred into $1 \mathrm{ml}$ of TRIzol ${ }^{\mathrm{TM}}$ LS reagent for RNA isolation at the same time points already defined for the ex vivo skin explants up to $18 \mathrm{~h}$.

\section{RNA Isolation, Reverse Transcription and Quantitative Real-Time PCR}

Bacteria were lysed and RNA isolation was performed as described previously (Burian et al., 2010a). To eliminate contaminating DNA each RNA sample was digested with $8 \mathrm{U}$ of RNase-free DNase I (Roche), $2 \mu 110 \times$ incubation buffer (Roche), and $16 \mathrm{U}$ of RNasin ribonuclease inhibitor (Promega) for $30 \mathrm{~min}$ at $25^{\circ} \mathrm{C}$. DNase treatment was carried out twice for each sample. DNase I treatment was stopped using DNase inactivation reagent (Thermo Fisher Scientific). Three microliter of total RNA were transcribed into cDNA using Super Script III Reverse Transcriptase (Thermo Fisher Scientific) and 200 ng of random hexamer primers (Thermo Fisher Scientific). Reverse transcription was performed as described in the instructions of the Superscript manufacturer. cDNAs were diluted 1:3 with nuclease-free water (Thermo Fisher Scientific) and frozen at $-20^{\circ} \mathrm{C}$ using Eppendorf LoBind Tubes (Eppendorf, Hamburg, Germany) for prolonged storage.

Relative quantification of $S$. aureus transcripts by qPCR was carried out using the 7300 Real Time PCR instrument (Applied Biosystems) in combination with the KAPA SYBR ${ }^{\circledR}$ FAST qPCR Master Mix (2x) ABI Prism (Merck). Master mixes were prepared as following: $8 \mu \mathrm{l} \mathrm{KAPA} \mathrm{SYBR}{ }^{\circledR}$ FAST qPCR Master Mix $(2 \mathrm{x})$ ABI Prism, $8 \mu \mathrm{l}$ nuclease-free water, $1 \mu \mathrm{l}$ of each primer (see Supplementary Table 1; Burian et al., 2010a,b), and $2 \mu \mathrm{l}$ cDNA. The following temperature profile was utilized for amplification: denaturation for 1 cycle at $95^{\circ} \mathrm{C}$ for $15 \mathrm{~s}$ and 55 cycles at $95^{\circ} \mathrm{C}$ for $27 \mathrm{~s}, 55-60^{\circ} \mathrm{C}$ for $10 \mathrm{~s}$, and $72^{\circ} \mathrm{C}$ for $27 \mathrm{~s}$ with fluorescence acquisition at $72^{\circ} \mathrm{C}$. Melting-curve analysis was done at $60-97^{\circ} \mathrm{C}$ with stepwise fluorescence acquisition. Relative quantities of transcripts were calculated by a standard curve for each gene generated using sixfold serial dilution of $S$. aureus USA300 wild type RNA.

\section{Skin Explant Viability by Membrane-Permeable Dye Thiazolyl Blue Tetrazolium Bromide Assay}

The membrane-permeable dye thiazolyl blue tetrazolium bromide (MTT) was used to assess skin explant viability. Four millimeter skin biopsies were cultured as described above up to 10 days. From day 5 onward skin explants were washed twice with PBS and further incubated in $2 \mathrm{mg} / \mathrm{ml}$ MTT (SigmaAldrich) for $2 \mathrm{~h}$ at $37^{\circ} \mathrm{C}$ and $5 \% \mathrm{CO}_{2}$. MTT is reduced from yellow color to purple formazan in living skin biopsies.

\section{TUNEL Assay}

Apoptosis was assessed on skin tissue using an in situ Apoptosis Detection Kit (Sigma-Aldrich) according to the manufacturer's protocol. After the final washing step, sections were mounted in ibidi Mounting Medium with DAPI (ibidi). Staining was visualized using a Leica DMI4000 B microscope (Leica).

\section{Histological Analysis and Immunostaining}

Hematoxylin and eosin (H\&E) staining, as well as immunostaining, were carried out as previously described by Huth et al. (2018). Briefly, for immunofluorescence, 4 $\mu \mathrm{m}$ cryosections were fixed for $10 \mathrm{~min}$ in acetone at $4^{\circ} \mathrm{C}$. First antibodies keratin 10 (Dako) and filaggrin (AKH1) (Santa Cruz) were diluted with Antibody Diluent (Dako) and incubated at room temperature for $1 \mathrm{~h}$. Following washing steps with PBS, the sections were incubated in fluorochromeconjugated secondary antibody Alexa Fluor 488 IgG $\mathrm{H}+\mathrm{L}$ (Molecular Probes) for $1 \mathrm{~h}$ at room temperature. Cell nuclei were stained with DAPI (Applichem). All image processing and analyses were performed with ImageJ software (National Institutes of Health).

\section{Statistical Analysis}

Statistical analysis was performed with the Prism 9.0.2 package (GraphPad Software) using multiple $t$-tests. $P<0.05$ was considered to be statistically significant.

\section{RESULTS}

\section{Viability of Skin Explants}

Healthy human skin tissue was cultured at the air-liquid interface for up to 10 days (Figure 1A). To assess the viability of the skin explants we analyzed the ability of the tissue to reduce the MTT. Reduction from yellow color to purple formazan in living skin biopsies was observed for up to 8 days. At later time points, the cell viability decreased in skin explants (Figure 1B). In addition, we performed a validation of the integrity of our skin explants following the validation described by Neil et al. (2020). Using H\&E stained slides, neither signs of spongiosis, necrosis or cell death could be observed. The epidermis and dermis are not separated over this period of time and no alterations in the corneal layer were observed. Furthermore, the terminal differentiation marker filaggrin and keratin 10 were unaffected during this period (Supplementary Figure 2). We decided to follow the $S$. aureus adaptation process for up to day 8. During this period, we confirmed stable colonization of the skin samples with $S$. aureus by demonstrating persistent expression levels of the house keeping gene gyrB (Figure 1C). gyrB transcripts were stable over time, both from skin samples that were colonized with exponentially grown bacteria as well as from skin samples colonized with post exponentially grown bacteria (mean gyrB exponential bacteria $2.20 \times 10^{4}$ and mean gyr $\mathrm{B}$ post exponential bacteria $\left.1.60 \times 10^{4}\right)$. As expected, gyrB values of bacteria grown in vitro (medium only without skin) increased over time, indicating a growth in the cell culture medium (Figure 1C). 


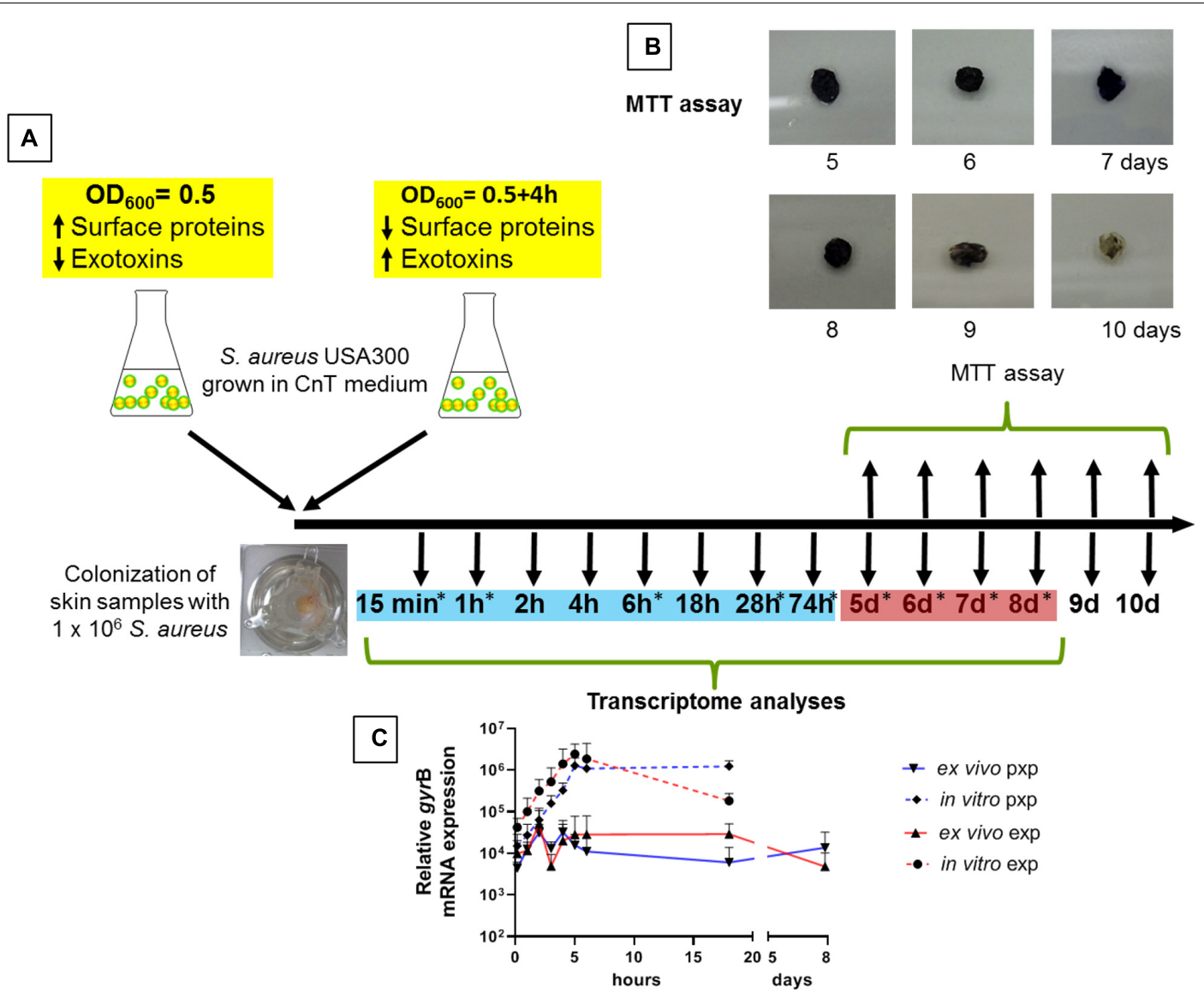

FIGURE 1 | Skin colonization model and experimental time course. (A) To analyze the adaptation of S. aureus to the epidermis, skin explants were colonized with either exponentially or post-exponentially grown S. aureus USA300 and transcript analyzes were performed up to $18 \mathrm{~h}$. For a broad screening of genes, skin samples were colonized with exponentially grown bacteria and transcript analyses were carried out at 9 different times indicated with an asterisk. Time points marked in blue are defined as early colonization and red marked times are defined as late colonization. (B) Skin explant viability by MTT assay. A reduction in color from yellow color to purple formazan has been observed in living skin biopsies for up to 8 days. (C) Relative gyrB mRNA expression over time. Exp, exponential phase; pxp, post-exponential phase.

\section{S. aureus Colonization of Human Skin Results in Uniform agr Inhibition Independent of the Inoculum Growth Status}

To profile the expression pattern of $S$. aureus during skin colonization we performed transcript analysis by quantitative real-time PCR (qPCR) starting 15 min after initial colonization. To evaluate, whether contact with the epidermis induced a uniform change in the expression pattern independently of the initial transcription level, we colonized skin samples with either exponentially or post-exponentially grown S. aureus (Figure 1A). In addition, wells containing medium only, were also inoculated with the corresponding inoculum to analyze gene expression during in vitro growth in the absence of skin contact. Initially, we analyzed transcription of RNAIII of the quorum sensing system $a g r$, which is usually repressed during exponential growth, and transcription of clfB as marker for an exponentially expressed gene (Figure 2). Strikingly, agr transcription was immediately switched off upon bacterial contact to the skin surface. Notably, the massive agr downregulation also took place when skin samples were colonized with post-exponentially grown bacteria in which agr is highly active (Figure 2A). Thus, contact with skin led to a downregulation of agr in post-exponentially and exponentially grown bacteria and this downregulation lasted for up to $18 \mathrm{~h}$. In contrast to agr suppression, transcription of $c l f \mathrm{~B}$ was induced in post exponentially grown bacteria upon skin contact or remained high when inoculated with expontential grown bacteria (Figure 2B). In summary, S. aureus contact with human skin results in consistent transcriptional changes independent of the inoculum's growth phase.

\section{Comparative Analysis of Bacterial Gene Expression Between ex vivo Co-culture and in vivo Colonization of the Nasal Cavity}

Subsequently, we analyzed the expression of $21 \mathrm{~S}$. aureus genes associated with a variety of cellular functions such as virulence regulation, toxin production, adhesion, immune evasion and cell 

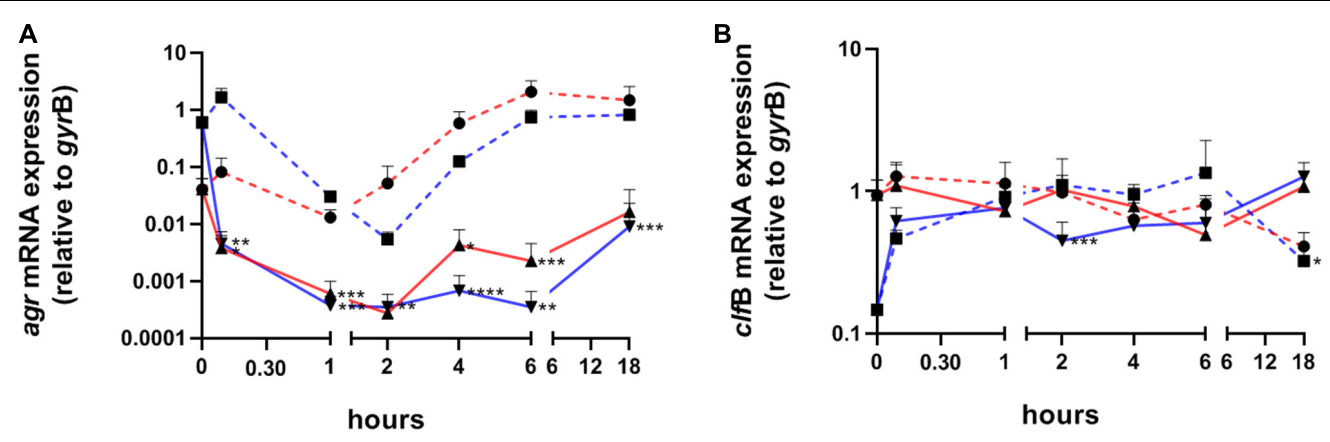

FIGURE 2 | Transcriptional analysis of $S$. aureus colonizing human skin samples after exponential or post-exponential growth. Transcripts were quantified in reference to the transcription of gyrB in samples colonized with exponentially (red lines) or post-exponentially grown $S$. aureus (blue lines). Dashed lines represent growth in vitro (without skin and medium only). Values are the mean value of at least 3 colonized skin samples from different individuals. (A) Relative transcription of the accessory gene regulator (agr) and (B) of clumping factor B (clfB). Statistically significant differences between exponential or post-exponentially ex vivo samples and their corresponding in vitro samples were indicated. ${ }^{\star} P \leq 0.05 ;{ }^{* \star} P \leq 0.01 ;{ }^{\star \star \star} P \leq 0.001 ;{ }^{* \star \star \star} P \leq 0.0001$.

wall dynamics. The expression of these genes during colonization of the human nasal cavity was previously assessed (Burian et al., 2010b) and was used for comparison (Figure 3). Skin explants were colonized with exponentially grown bacteria and gene expression was followed over 8 days during the colonization process. To define early and late colonization, time points up to $74 \mathrm{~h}$ were defined as early and $5-8$ days as late colonization (Figure 1A). The results are summarized according to the functional categorization of target genes.

\section{Global Regulators}

In $S$. aureus a complex regulatory network is responsible for differential gene expression resulting in a characteristic in vitro pattern- high adhesion expression in the early stage of growth and high toxin production during the later stages (Cheung et al., 2004). We investigated the activity of five well-defined regulators: $a g r, s a e, s i g \mathrm{~B}$ (detected as the tightly sigB-dependent gene asp23), graRS, and walKR. The massive downregulation of $a g r$ described above persisted over the entire period of colonization (Figure 3A). Expression of the twocomponent systems sae and walKR was not influenced by skin colonization (Figure $\mathbf{3 A}$ and Supplementary Figure 1A). In contrast, expression of sigB and graRS was upregulated after contact with skin, especially during late colonization. Here, graRS transcription even exceeded maximal transcription levels in vitro, suggesting a role of these two regulators during skin colonization (Figure 3A).

\section{Toxins}

Toxins such as the alpha-hemolysin ( $h l a)$ or the phenol-soluble modulins $(p s m)$ are highly expressed during post-exponential growth as they are positively regulated by the agr system (Queck et al., 2008; Jenul and Horswill, 2019). In accordance to agr transcription, a massive downregulation was observed immediately after bacterial contact to the skin (Figure 3A).

\section{Adhesins}

S. aureus cell-wall-anchored proteins and other adhesive molecules are implicated in binding to host matrix molecules and may therefore be critical during the colonization process. Interestingly, the fibrinogen binding protein clumping factor A (clf A) and the fibrinogen and fibronectin binding protein fnbA, both sigB target genes (Goerke and Wolz, 2004), were strongly upregulated upon skin contact. Transcription of clf A remained constantly high over time and even exceeded the maximal transcription observed in vitro (Figure $\mathbf{3 B}$ and Supplementary Figure 1B).

S. aureus wall teichoic acid (WTA) is an important colonization factor mediating adherence to epithelial and endothelial cells (Weidenmaier and Peschel, 2008). We characterized the role of WTA during skin colonization by analyzing the transcription of the WTA biosynthesis gene $\operatorname{tag} \mathrm{O}$. In our study tagO transcription between day 6 and 8 constantly exceeded maximal in vitro transcription (Figure 3B).

\section{Immune Evasion and Immune Modulatory Factors}

Immune evasion is a prerequisite for stable and sustained colonization. In S. aureus it is mediated by a large number of molecules, such as protein A (spa), the extracellular capsular polysaccharide (cap) and the prophage encoded immune evasion molecules staphylokinase (sak), staphylococcal complement inhibitor $(s c n)$ and chemotaxis inhibitory protein (chp). Transcript analysis of spa and cap revealed low expression levels whereas transcription of $s c n$ and sak was increased during late colonization (Figure $\mathbf{3 B}$ and Supplementary Figure 1B).

\section{Cell-Wall Modification Enzymes}

Enzymes involved in cell wall remodeling contribute to resistance against antimicrobial peptides and modulate the immune response. Expression of oat A, dltA, and $m p r F$ was only slightly altered upon skin contact. However, expression of sce D and atlA was highly induced throughout the course of skin colonization (Figure 3C).

In summary, the comparison between ex vivo skin co-culture and in vivo nose colonization revealed similar changes in gene expression for most genes as for example the strong inhibition of the agr system and the down regulation of genes encoding toxins 

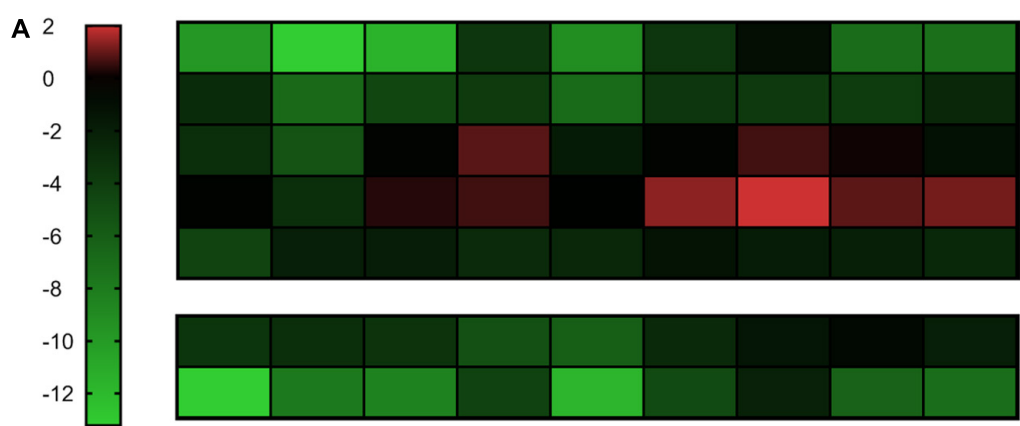
agr
sae
asp23 (sigB)
graRS
walKR
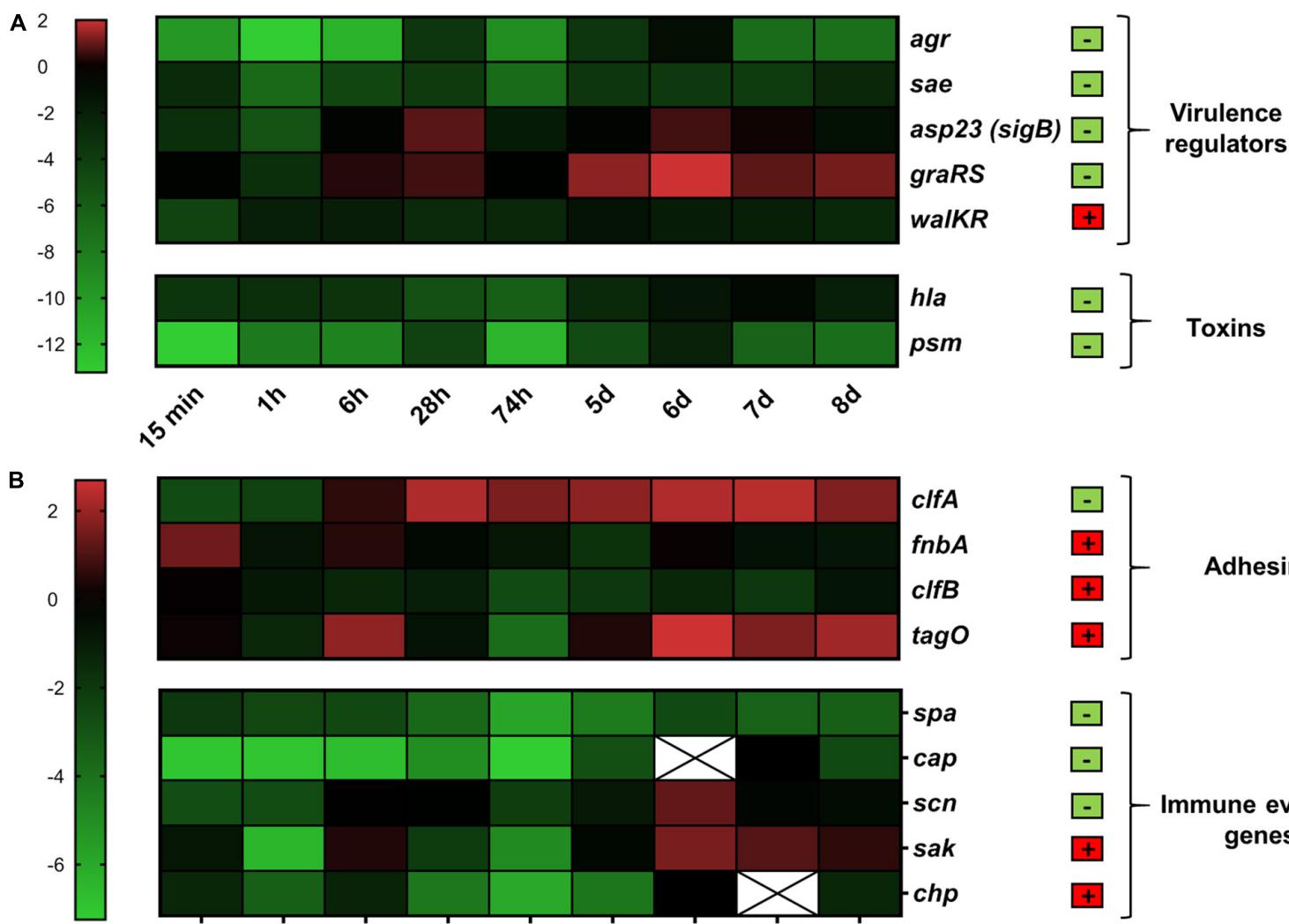

Adhesins
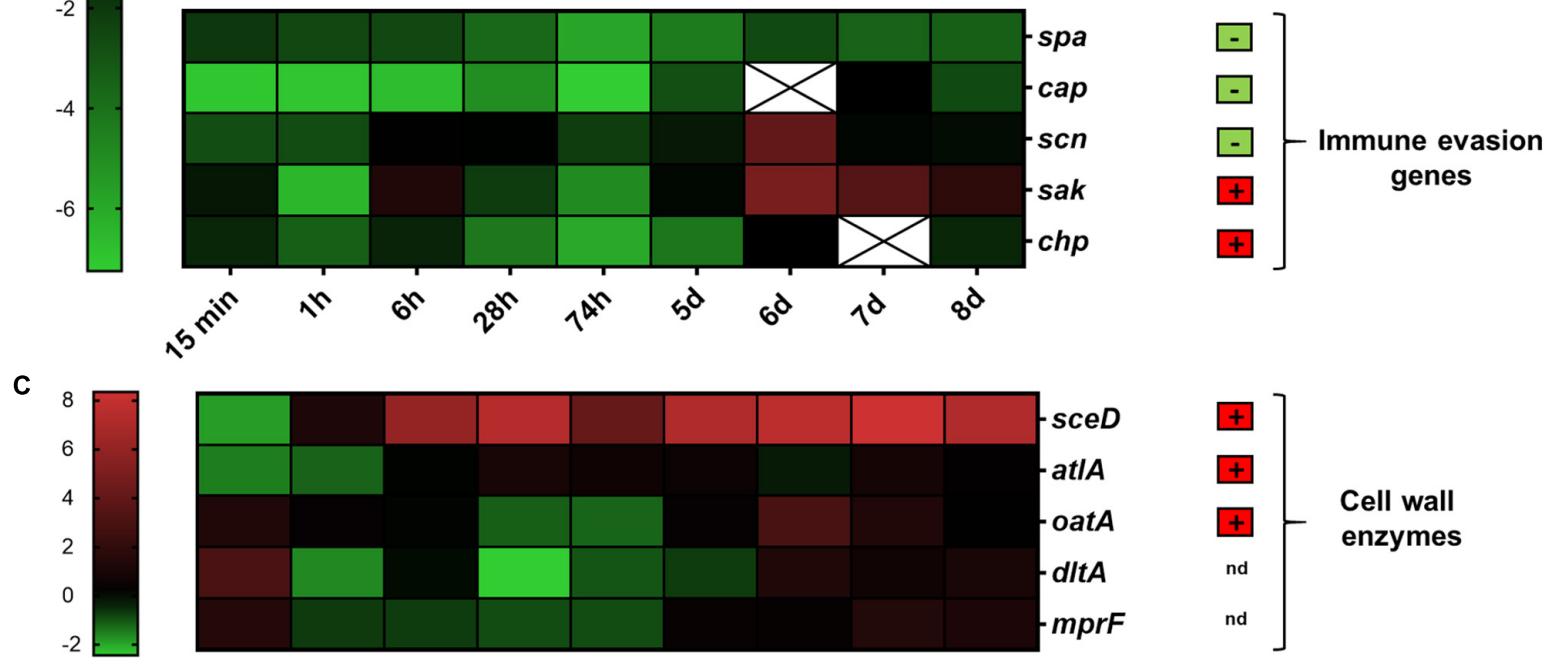

FIGURE 3 | Transcriptional analysis during human skin colonization. Results are depicted as the ratio of transcription ex vivo vs. maximal expression in vitro. All data were log transformed (basis 2) and changes in gene expression were normalized in reference to the constitutively expressed gene gyrB. Genes colored red were up-regulated compared to in vitro; genes colored green were down-regulated compared to in vitro. Black indicates the same expression levels ex vivo and in vitro. White cells with an " $x$ " have no values because gene expression was below the detection limit. Results are the mean values of 3 different skin samples from different individuals. (A) Genes belonging to virulence regulation and toxin production were clustered. (B) Genes involved in adhesion and immune evasion were combined. (C) Genes involved in cell wall modification. Genes relevant during nasal colonization were marked with a + (box with red background), while genes that were not relevant during nasal colonization were marked with a - (box with green background) (Burian et al., 2010b). Gene name abbreviations see Supplementary Table 2. The color chart was generated using GraphPad Prism 9.0.2.

or the high induction of $s c e \mathrm{D}$. However, the strong induction of clf A and graRS seems to be specific for skin colonization.

\section{Proteases of All Three Catalytic Classes Were Strongly Transcribed Immediately After Contact of $S$. aureus With Human Skin}

The data support that toxicity of S. aureus is dampened through inactivation of virulence regulators such as agr. Moreover, toxins might be inactivated through proteolytic cleavage (Gimza et al., 2021). On the other hand S. aureus secreted proteases might also contribute to tissue degradation and inactivation of host defense systems (Singh and Phukan, 2019). For Staphylococcus epidermidis the protease EcpA can be a deleterious component of the skin microbiome in $\mathrm{AD}$ (Cau et al., 2021). Therefore, we subsequently focused on the analysis of genes coding for major proteases: the metalloprotease aureolysin (aur), the serine proteases sspA (V8 protease), and splA (member of the protease-like proteins), and the cysteine proteases staphopain A and B (encoeded by $s c p \mathrm{~A}$ and $s s p \mathrm{~B}$ ). Transcription of all proteases was strongly induced during the 

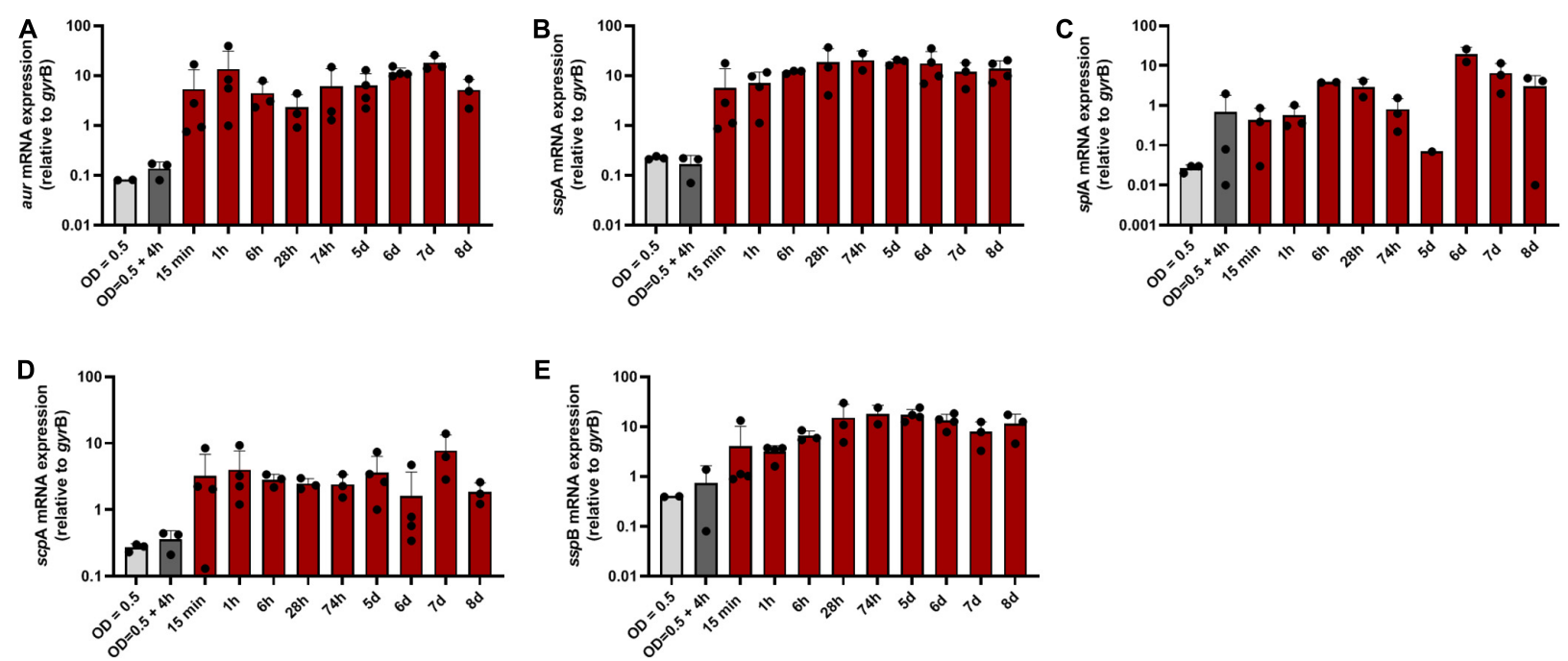

FIGURE 4 | Transcription of protease-encoding genes during colonization of human skin. Transcripts were quantified in reference to the transcription of gyrB in skin samples colonized with S. aureus. (A) Relative transcription of the metalloprotease aureolysin (aur), the serine proteases sspA (V8 protease) (B) and splA (C), and the cysteine proteases staphopain A and B [scpA (D) and sspB (E)].

entire time period of colonization starting already after $15 \mathrm{~min}$ with the exception of splA, which was transcribed at low levels and peaked only during late colonization (Figure 4).

\section{DISCUSSION}

S. aureus is able to interact with its human host, both as a pathogen and as a commensal. The knowledge of bacterial gene regulation upon epidermal contact and the circumstances influencing gene regulation during skin colonization are crucial for understanding their role in infection. In the present study, we investigated $S$. aureus adaption to human skin using ex vivo skin explants. This model allowed us to longitudinally follow the colonization and the virulence factor expression in $S$. aureus as response to the contact with human skin tissue. Here, a differentiated expression pattern reflecting the adaptation to the epidermis was observed during early colonization. Notably, this response was independent of the growth phase of the S. aureus inoculum.

Most prominently, skin contact resulted in immediate inhibition of the quorum sensing system Agr. Of note, agr transcription was even lower than the expression in bacteria at the early exponential growth phase known to suppress the Agr system. Thus, the inhibition of agr expression can only in part be explained by culture dilution. Consistent with low agr transcription, typical agr target genes ( $h l a, c a p, p s m$ ) remained downregulated during skin exposure. Furthermore, the low agr transcription as well as the downregulation of agr target genes is consistent with the expression profile found during nasal colonization (Burian et al., 2010a,b). The strict shutdown of agr immediately upon contact to the skin indicates that at least during early colonization agr inactivation promotes bacterial colonization. On the native skin other staphylococcal species may further contribute to agr inhibition since some of them produce agr autoinducing molecules, which competitively inhibit the Agr system of S. aureus (Jenul and Horswill, 2019). Nevertheless, the global virulence regulator Agr and in particular the Agr dependent PSMs are known to significantly contribute to skin diseases such as AD (Williams et al., 2019) or abscess formation (Wright et al., 2005). One may speculate that Agr becomes activated once the bacteria breaches through the intact barrier of the skin. The mechanisms leading to this postulated Agr activation remain to be elucidated and will be the subject of future investigations.

In opposition to asymptomatic colonization of the nose, where WalKR seems to be one major regulator for adaptive gene expression (Burian et al., 2010b), S. aureus adaptation to the skin was correlated with activation of the antimicrobial peptide sensing system graRS and the alternative sigma factor $\mathrm{B}$. In accordance to sig $\mathrm{B}$ activity, expression of the sig $\mathrm{B}$ target genes $c l f \mathrm{~A}$ and $f n b \mathrm{~A}$ (Goerke and Wolz, 2004) was also induced upon skin contact suggesting a role during adhesion. Thus, the gene expression profile during colonization of the skin differs partly from that during colonization of the human nose, especially in terms of global regulatory elements and in adhesion. Nevertheless, there are also remarkable similarities between skin and nasal colonization, for example the high transcription of the autolysins sce D and atlA (Burian et al., 2010b).

In addition to adhesion and cell wall remodeling, another essential step in colonization of the skin is the defense against the host's immune response. In $S$. aureus immune evasion is mediated by a large number of molecules (Foster, 2005) including proteases. Besides their function in tissue degradation, interception of host enzymes and bacterial adhesion regulation (Dubin, 2003), proteases are able to inactivate antimicrobial peptides (Sieprawska-Lupa et al., 2004). For example, aureolysin and staphopain cleave and inactivate the human defensin peptide 
LL-37, which is involved in AD (Sieprawska-Lupa et al., 2004; Foster, 2005; Sonesson et al., 2017). Since both proteases were highly transcribed throughout the colonization process they might protect $S$. aureus from antimicrobial peptides and contribute to successful colonization. Interestingly, none of the skin colonizing bacteria can actively penetrate intact skin (Koziel and Potempa, 2013). Prerequisite for skin penetration is a defect of the epidermal integrity for example by small cuts. Therefore, it would be interesting to analyze the role of proteases during the switch from colonization to infection in an impaired skin barrier model, a possible scenario using our newly introduced ex vivo skin model (Marquardt et al., 2015).

\section{CONCLUSION AND OUTLOOK}

By using our skin explant colonization model, we provide first insights into the adaptation strategy of $S$. aureus during the early phase of human skin colonization. We demonstrated that skin explants as well the inoculated $S$. aureus strain can survive over several days, providing a model to mimic asymptomatic carriage of $S$. aureus on healthy skin. The model will allow in the future to unravel the molecular mechanism of gene regulation under these specific conditions at the air-liquid interface and to obtain a more comprehensive picture of gene expression by for example RNAseq analysis. There are two major limitations in this study. First, the adaptation of $S$. aureus was evaluated neglecting the skin's microbiota, which should be considered in future studies, and second, due to technical limitations, information on protein level could not be provided. From the preliminary data one can speculate that $S$. aureus is equipped with a yet not described "contact sensing system." Moreover, the model should allow the analysis of bacterial interference over time and the impact of host immune factors such as cytokines on bacteria. Although both the nose and the epidermis are colonized by the microbe, there are considerable differences in regulatory elements and adhesion depending on the organ colonized. But the two organs also share the induction of bacterial pathways, such as the strong transcription of $s c e \mathrm{D}$. These common regulatory genes might be crucial for a successful decolonization strategy avoiding

\section{REFERENCES}

Alsterholm, M., Strombeck, L., Ljung, A., Karami, N., Widjestam, J., Gillstedt, M., et al. (2017). Variation in Staphylococcus aureus colonization in relation to disease severity in adults with atopic dermatitis during a five-month follow-up. Acta Derm. Venereol. 97, 802-807. doi: 10.2340/00015555-2667

Burian, M., Grumann, D., Holtfreter, S., Wolz, C., Goerke, C., and Broker, B. M. (2012). Expression of staphylococcal superantigens during nasal colonization is not sufficient to induce a systemic neutralizing antibody response in humans. Eur. J. Clin. Microbiol. Infect. Dis. 31, 251-256. doi: 10.1007/s10096-011-13022

Burian, M., Rautenberg, M., Kohler, T., Fritz, M., Krismer, B., Unger, C., et al. (2010a). Temporal expression of adhesion factors and activity of global regulators during establishment of Staphylococcus aureus nasal colonization. J. Infect. Dis. 201, 1414-1421. doi: 10.1086/651619 symptomatic skin infections. Virulence factors with significant expression in different body habitats might provide new targets for specific therapy and prevention.

\section{DATA AVAILABILITY STATEMENT}

The raw data supporting the conclusions of this article will be made available by the authors, without undue reservation.

\section{ETHICS STATEMENT}

The studies involving human participants were reviewed and approved by the Ethics Commission at the Medical Faculty of RWTH University Aachen, Pauwelsstr. 30, 52074 Aachen, Germany. The patients/participants provided their written informed consent to participate in this study.

\section{AUTHOR CONTRIBUTIONS}

$\mathrm{MB}, \mathrm{CW}$, and AY performed the study concept and design. MB, $A K, Y M$, and $A Y$ designed and performed the experiments. $M B$, $\mathrm{JB}, \mathrm{MH}, \mathrm{CW}$, and AY wrote the manuscript. MB, JP, LS, AK, LH, $\mathrm{CW}$, and AY analyzed the data. All authors contributed to the article and approved the submitted version.

\section{FUNDING}

This work was supported by a grant from the START-Program of the Faculty of Medicine of the RWTH Aachen University and by CRC TRR 156 by the DFG (to CW and AY).

\section{SUPPLEMENTARY MATERIAL}

The Supplementary Material for this article can be found online at: https://www.frontiersin.org/articles/10.3389/fmicb. 2021.728989/full\#supplementary-material

Burian, M., Wolz, C., and Goerke, C. (2010b). Regulatory adaptation of Staphylococcus aureus during nasal colonization of humans. PLoS One 5:e10040. doi: 10.1371/journal.pone.0010040

Cau, L., Williams, M. R., Butcher, A. M., Nakatsuji, T., Kavanaugh, J. S., Cheng, J. Y., et al. (2021). Staphylococcus epidermidis protease EcpA can be a deleterious component of the skin microbiome in atopic dermatitis. J. Allergy Clin. Immunol. 147:e916.

Chen, Y. E., Fischbach, M. A., and Belkaid, Y. (2018). Skin microbiota-host interactions. Nature 553, 427-436. doi: 10.1038/nature25177

Cheung, A. L., Bayer, A. S., Zhang, G., Gresham, H., and Xiong, Y. Q. (2004). Regulation of virulence determinants in vitro and in vivo in Staphylococcus aureus. FEMS Immunol. Med. Microbiol. 40, 1-9. doi: 10.1016/s0928-8244(03) 00309-2

Dubin, G. (2003). Defense against own arms: staphylococcal cysteine proteases and their inhibitors. Acta Biochim. Pol. 50, 715-724. doi: 10.18388/abp.2003_3662 
Fey, P. D., Endres, J. L., Yajjala, V. K., Widhelm, T. J., Boissy, R. J., Bose, J. L., et al. (2013). A genetic resource for rapid and comprehensive phenotype screening of nonessential Staphylococcus aureus genes. mBio 4:e00537-12.

Foster, T. J. (2005). Immune evasion by staphylococci. Nat. Rev. Microbiol. 3, 948-958. doi: 10.1038/nrmicro1289

Gimza, B. D., Jackson, J. K., Frey, A. M., Budny, B. G., Chaput, D., Rizzo, D. N., et al. (2021). Unraveling the impact of secreted proteases on hypervirulence in Staphylococcus aureus. mBio 12:e03288-20. doi: 10.1128/mBio.03288-20

Goerke, C., and Wolz, C. (2004). Regulatory and genomic plasticity of Staphylococcus aureus during persistent colonization and infection. Int. J. Med. Microbiol. 294, 195-202. doi: 10.1016/j.ijmm.2004.06.013

Huth, S., Schmitt, L., Marquardt, Y., Heise, R., Lüscher, B., Amann, P. M., et al. (2018). Effects of a ceramide-containing water-in-oil ointment on skin barrier function and allergen penetration in an IL-31 treated 3D model of the disruped skin barrier. Exp. Dermatol. 27, 1009-1014. doi: 10.1111/exd.13697

Jenul, C., and Horswill, A. R. (2019). Regulation of Staphylococcus aureus virulence. Microbiol. Spectr. 7:10.1128/microbiolspec.GPP3-0031-2018.

Koziel, J., and Potempa, J. (2013). Protease-armed bacteria in the skin. Cell Tissue Res. 351, 325-337. doi: 10.1007/s00441-012-1355-2

Marquardt, Y., Amann, P. M., Heise, R., Czaja, K., Steiner, T., Merk, H. F., et al. (2015). Characterization of a novel standardized human three-dimensional skin wound healing model using non-sequential fractional ultrapulsed CO2 laser treatments. Lasers Surg. Med. 47, 257-265. doi: 10.1002/lsm.22341

Matsui, K., Nishikawa, A., Suto, H., Tsuboi, R., and Ogawa, H. (2000). Comparative study of Staphylococcus aureus isolated from lesional and non-lesional skin of atopic dermatitis patients. Microbiol. Immunol. 44, 945-947. doi: 10.1111/j. 1348-0421.2000.tb02587.x

Neil, J. E., Brown, M. B., and Williams, A. C. (2020). Human skin explant model for the investigation of topical therapeutics. Nat. Sci. Rep. 10:21192.

Olaniyi, R., Pozzi, C., Grimaldi, L., and Bagnoli, F. (2017). Staphylococcus aureusassociated skin and soft tissue infections: anatomical localization, epidemiology, therapy and potential prophylaxis. Curr. Top. Microbiol. Immunol. 409, 199227. doi: 10.1007/82_2016_32

Queck, S. Y., Jameson-Lee, M., Villaruz, A. E., Bach, T. H., Khan, B. A., Sturdevant, D. E., et al. (2008). RNAIII-independent target gene control by the agr quorum-sensing system: insight into the evolution of virulence regulation in Staphylococcus aureus. Mol. Cell 32, 150-158. doi: 10.1016/j.molcel.2008.08.005

Shi, B., Bangayan, N. J., Curd, E., Taylor, P. A., Gallo, R. L., Leung, D. Y. M., et al. (2016). The skin microbiome is different in pediatric versus adult atopic dermatitis. J. Allergy Clin. Immunol. 138, 1233-1236. doi: 10.1016/j.jaci.2016. 04.053

Sieprawska-Lupa, M., Mydel, P., Krawczyk, K., Wojcik, K., Puklo, M., Lupa, B., et al. (2004). Degradation of human antimicrobial peptide LL-37 by
Staphylococcus aureus-derived proteinases. Antimicrob. Agents Chemother. 48, 4673-4679. doi: 10.1128/aac.48.12.4673-4679.2004

Singh, V., and Phukan, U. J. (2019). Interaction of host and Staphylococcus aureus protease-system regulates virulence and pathogenicity. Med. Microbiol. Immunol. 208, 585-607. doi: 10.1007/s00430-018-0 573-y

Sonesson, A., Przybyszewska, K., Eriksson, S., Morgelin, M., Kjellstrom, S., Davies, J., et al. (2017). Identification of bacterial biofilm and the Staphylococcus aureus derived protease, staphopain, on the skin surface of patients with atopic dermatitis. Sci. Rep. 7:8689.

Totte, J. E., Van Der Feltz, W. T., Hennekam, M., Van Belkum, A., Van Zuuren, E. J., and Pasmans, S. G. (2016). Prevalence and odds of Staphylococcus aureus carriage in atopic dermatitis: a systematic review and meta-analysis. Br. J. Dermatol. 175, 687-695. doi: 10.1111/bjd.14566

Weidenmaier, C., and Peschel, A. (2008). Teichoic acids and related cell-wall glycopolymers in Gram-positive physiology and host interactions. Nat. Rev. Microbiol. 6, 276-287. doi: 10.1038/nrmicro1861

Williams, M. R., Costa, S. K., Zaramela, L. S., Khalil, S., Todd, D. A., Winter, H. L., et al. (2019). Quorum sensing between bacterial species on the skin protects against epidermal injury in atopic dermatitis. Sci. Transl. Med. 11:eaat8329. doi: 10.1126/scitranslmed.aat8329

Wright, J. S. III, Jin, R., and Novick, R. P. (2005). Transient interference with staphylococcal quorum sensing blocks abscess formation. Proc. Natl. Acad. Sci. U S A. 102, 1691-1696. doi: 10.1073/pnas.040766 1102

Conflict of Interest: The authors declare that the research was conducted in the absence of any commercial or financial relationships that could be construed as a potential conflict of interest.

Publisher's Note: All claims expressed in this article are solely those of the authors and do not necessarily represent those of their affiliated organizations, or those of the publisher, the editors and the reviewers. Any product that may be evaluated in this article, or claim that may be made by its manufacturer, is not guaranteed or endorsed by the publisher.

Copyright (c) 2021 Burian, Plange, Schmitt, Kaschke, Marquardt, Huth, Baron, Hornef, Wolz and Yazdi. This is an open-access article distributed under the terms of the Creative Commons Attribution License (CC BY). The use, distribution or reproduction in other forums is permitted, provided the original author(s) and the copyright owner(s) are credited and that the original publication in this journal is cited, in accordance with accepted academic practice. No use, distribution or reproduction is permitted which does not comply with these terms. 